\title{
Short-term blood pressure variability - variation between arm side, body position and successive measurements: a population-based cohort study
}

Maria Elena Lacruz ${ }^{1 * \dagger}$, Alexander Kluttig ${ }^{1 \dagger}$, Oliver Kuss ${ }^{2,3}$, Daniel Tiller ${ }^{1}$, Daniel Medenwald ${ }^{1}$, Sebastian Nuding ${ }^{4}$, Karin Halina Greiser ${ }^{5}$, Stefan Frantz ${ }^{4}$ and Johannes Haerting ${ }^{1}$

\begin{abstract}
Background: Precise blood pressure (BP) measurements are central for the diagnosis of hypertension in clinical and epidemiological studies. The purpose of this study was to quantify the variability in BP associated with arm side, body position, and successive measurements in the setting of a population-based observational study. Additionally, we aimed to evaluate the influence of different measurement conditions on prevalence of hypertension.

Methods: The sample included 967 men and 812 women aged 45 to 83 years at baseline. BP was measured according to a standardized protocol with oscillometric devices including three sitting measurements at left arm, one simultaneous supine measurement at both arms, and four supine measurements at the arm with the higher BP. Hypertension was defined as systolic BP (SBP) $\geq 140 \mathrm{mmHg}$ and/or diastolic BP (DBP) $\geq 90 \mathrm{mmHg}$. Variability in SBP and DBP were analysed with sex-stratified linear covariance pattern models.

Results: We found that overall, no mean BP differences were measured according to arm-side, but substantial higher DBP and for men also higher SBP was observed in sitting than in supine position and there was a clear BP decline by consecutive measurement. Accordingly, the prevalence of hypertension depends strongly on the number and scheme of BP measurements taken to calculate the index values.

Conclusions: Thus, BP measurements should only be compared between studies applying equal measurement conditions and index calculation. Moreover, the first BP measurement should not be used to define hypertension since it overestimates BP. The mean of second and third measurement offers the advantage of better reproducibility over single measurements.
\end{abstract}

Keywords: (3-5) arm-side, Blood pressure variability, Body position, Hypertension, Successive measurements

\section{Background}

The indirect blood pressure (BP) measurement by a trained observer is widely used in clinical and epidemiological practice as accurate BP measurement. Office BP has shown a good validity when compared with 24-h ambulatory BP measurements (correlation of 0.9 and 0.8 for systolic BP and diastolic BP respectively) [1]. Precise

\footnotetext{
* Correspondence: elena.lacruz@uk-halle.de

${ }^{\dagger}$ Equal contributors

${ }^{1}$ Institute of Medical Epidemiology, Biostatistics and Informatics,

Martin-Luther University Halle-Wittenberg, Magdeburger Str. 8, 06112 Halle

Saale, Germany

Full list of author information is available at the end of the article
}

BP measurements are central for the diagnosis of hypertension and for clinical or epidemiological studies where blood pressure is used as exposure or outcome of interest.

Several factors affect BP measurement, such as environmental factors (i.e. temperature, noise); factors related to the participant (i.e. vigorous physical activity, heavy meal or smoking before measurement); factors related to the device (i.e. cuff size, calibration error) or to the measurement procedure (i.e. left vs. right arm, supine vs. sitting) [2-7]. According to Tolonen et al. [8], these result in variations from $1-2 \mathrm{mmHg}$ up to $20-50 \mathrm{mmHg}$ in individual measurements. 
Inter-arm differences in BP vary with the population studied. Some previous studies have suggested a bias towards higher readings from the right arm [9-11], whereas others have failed to show this [12-15]. Additionally, disagreement exists as to whether body position would make any difference in BP readings [6, 16-18]. Several studies have shown that mean supine systolic BP (SBP) is $2-3 \mathrm{mmHg}$ higher and diastolic BP (DBP) $3-5 \mathrm{mmHg}$ lower than sitting BP $[6,16,17]$. However, other studies suggest that posture is unlikely to have significant impact on BP readings $[18,19]$. Further, little is known about the magnitude of differences between consecutive BP readings in epidemiologic studies. The results of these studies are conflicting regarding the arm-side, body position and reading order in measured BP (successive measurements in and of itself). Moreover, not only additional unmeasured conditions, but also, already known participants characteristics could further contribute to these inconsistencies. For example, the well-known sex-specific differences in onset and rate of hypertension [20]. Thus an epidemiological study with a highly standardised study protocol, from a sex and age-stratified random sample, measurements conducted by a single trained qualified study nurse and a single weekly checked device could provide helpful information in explaining these uncertainties. Considering the important clinical and epidemiological implications from these differences; i.e., variations in measured $\mathrm{BP}$ could lead to variable diagnosis and bias in BP estimates and hypertension frequencies from epidemiological studies, it is important to determine the estimated effect of each of these conditions on the measured BP.

The purpose of this study was therefore, to quantify the variability in $\mathrm{BP}$ associated with: a) arm side: right vs left; b) body position: sitting vs supine and c) successive measurements: 1 to 8 ; in the setting of a population based observational study. Additionally, we aimed to evaluate the influence of different measurement conditions on prevalence of hypertension (defined as SBP above or equal 140 and/or DBP above or equal $90 \mathrm{mmHg}$ ).

\section{Methods}

\section{Participants and setting}

The CARLA-Study is a population based cohort study in an elderly population of the city of Halle (Saale) in eastern Germany. Study design and methods were described in detail elsewhere [21]. In brief, subjects were recruited as a random and representative sample from the population registry in a multi-stage process. At baseline 1779 subjects (46\% women) aged 45 to 83 years were examined between July 2002 and January 2006 (response rate 64\%). The study was in accordance with the declaration of Helsinki. All participants gave their written informed consent. The study was approved by the local ethic commission at the Medical Faculty of the Martin-Luther-University Halle-Wittenberg.

\section{Outcome}

Measurement of BP was conducted within the medical examination after a resting phase of at least five minutes. Systolic and diastolic BP were measured in a sitting position with an oscillometric device (OMRON HEM$705 \mathrm{CP}$, OMRON, Tokyo, Japan) three times on the left arm with a break of three minutes between measurements, one simultaneous supine measurement at both arms, and four supine measurements at the arm with the higher BP (see flow chart, Additional file 1: Figure S1).

Hypertension was within CARLA defined as mean systolic $\mathrm{BP}$ (SBP) equal or above $140 \mathrm{mmHg}$, and/or mean diastolic BP (DBP) above $90 \mathrm{mmHg}$ (defined as the mean of second and third measurement) following [22].

\section{Statistical analysis}

Bland-Altman plots were used to graphically illustrate the variability for the $8 \mathrm{BP}$ measurements. BP values between measurements were evaluated by sex-stratified linear covariance pattern models [23]. The following variables were entered into all of the models as fixed effects (confounders): arm-position (left-sitting, left-supine, right-supine), measurement (1 to 8), and their interaction. A residual ARMA $(1,1)$ (autoregressive movingaverage) process was included to account for the additional correlation of BP measurements in the time course within the same participant. All analyses were performed with SAS 9.3 (SAS Inc., Cary, NC, USA).

\section{Results}

A total of 1779 participants (46\% women) took part in the baseline examinations, comparisons could be done among 1743 participants (45\% women) with measurements in left and right arm; 1728 (46\% women) with measurements in sitting and supine positions and 1778 (46\% women) participants with first to third sitting measurements (Table 1). One participant refused to have any BP measurements. Further 36 participants had no supine BP measurements due to contraindication (i.e. thrombose, amputation) or declined participation and for further 15 participants the supine BP measurements had to be stopped before completion.

Figure 1 shows that there were no large mean differences in the BP measurements in left vs right arm. Mean differences were for SBP $0.05 \mathrm{mmHg}$ (95\% CI (confidence interval) $=-0.27$ to 0.38 ) and for DBP $-0.98 \mathrm{mmHg}$ (95\% CI $=-1.19$ to- 0.77 ). Though, approximately $10 \%$ of the participants had differences greater than $10 \mathrm{mmHg}$ for SBP and about $15 \%$ had differences greater than $5 \mathrm{mmHg}$ for DBP. These differences of $10 \mathrm{mmHg}$ for SBP and $5 \mathrm{mmHg}$ for DBP have been previously shown to be clinically relevant [24].

A further analysis involved the order of the BP measurement (successive measurements): first vs second 
Table 1 Baseline characteristics of the CARLA study population (2002-2006): distribution of cardiovascular risk factors and diseases

\begin{tabular}{|c|c|c|c|c|c|c|}
\hline & \multicolumn{3}{|c|}{ Women } & \multicolumn{3}{|l|}{ Men } \\
\hline & $\mathrm{N}$ & Median & Min; Q1; Q3; Max & $\mathrm{N}$ & Median & Min; Q1; Q3; Max \\
\hline Age (years) & 812 & 63.3 & $45.8 ; 54.9 ; 71.8 ; 83.4$ & 967 & 64.9 & $45.4 ; 55.8 ; 74.0 ; 83.3$ \\
\hline $\mathrm{SBP}(\mathrm{mmHg})$ & 812 & 138.0 & $89.0 ; 125.5 ; 155.0 ; 231.0$ & 966 & 145.5 & $93.0 ; 131.5 ; 158.5 ; 227.0$ \\
\hline $\mathrm{DBP}(\mathrm{mmHg})$ & 812 & 82.5 & 54.0; 76.0; 90.0; 122.0 & 966 & 85.5 & $52.5 ; 78.5 ; 93.0 ; 127.0$ \\
\hline Heart rate (1/min) & 778 & 67.0 & $39.4 ; 60.9 ; 73.4 ; 105.4$ & 893 & 65.7 & $43.9 ; 60.2 ; 74.7 ; 112.4$ \\
\hline Total cholesterol (mmol/l) & 807 & 5.7 & $1.4 ; 5.0 ; 6.4 ; 10.7$ & 960 & 5.3 & $2.1 ; 4.9 ; 5.9 ; 17.8$ \\
\hline HDL cholesterol (mmol/l) & 807 & 1.5 & $0.4 ; 1.3 ; 1.8 ; 3.4$ & 960 & 1.2 & $0.3 ; 1.0 ; 1.5 ; 3.0$ \\
\hline LDL cholesterol (mmol/l) & 801 & 3.4 & $0.9 ; 2.8 ; 4.0 ; 7.3$ & 940 & 3.1 & $0.9 ; 2.6 ; 3.7 ; 7.2$ \\
\hline Cholesterol/HDL ratio & 807 & 3.8 & $1.7 ; 3.0 ; 4.5 ; 9.7$ & 960 & 4.3 & $1.7 ; 3.5 ; 5.2 ; 13.0$ \\
\hline Triglycerides (mmol/l) & 807 & 1.4 & $0.2 ; 1.0 ; 1.9 ; 11.6$ & 960 & 1.7 & $0.4 ; 1.2 ; 2.5 ; 38.2$ \\
\hline Glucose (mmol/l) & 807 & 5.4 & $3.1 ; 5.1 ; 5.9 ; 19.8$ & 960 & 5.6 & $3.0 ; 5.2 ; 6.3 ; 21.5$ \\
\hline $\mathrm{HbA}_{1 \mathrm{c}}(\%)$ & 807 & 5.6 & $3.1 ; 5.3 ; 5.9 ; 12.0$ & 960 & 5.6 & $2.8 ; 5.3 ; 6.0 ; 11.7$ \\
\hline Body mass index $\left(\mathrm{kg} / \mathrm{m}^{2}\right)$ & 812 & 28.0 & $18.1 ; 24.7 ; 31.5 ; 53.7$ & 967 & 27.7 & $16.5 ; 25.4 ; 30.8 ; 43.3$ \\
\hline Waist hip ratio & 812 & 0.9 & $0.7 ; 0.8 ; 0.9 ; 1.2$ & 967 & 1.0 & $0.8 ; 1.0 ; 1.0 ; 1.2$ \\
\hline For current smokers: pack-years & 119 & 19.5 & $0.2 ; 10.6 ; 26.6 ; 61.0$ & 225 & 29.0 & $0.5 ; 18.0 ; 38.4 ; 85.1$ \\
\hline \multirow[t]{2}{*}{ Alcohol consumption gram/day } & 812 & 0 & $0 ; 0 ; 5.0 ; 60.5$ & 964 & 12.5 & $0 ; 2.5 ; 26.2 ; 113.6$ \\
\hline & $\mathrm{N}$ & Proportion & $95 \% \mathrm{Cl}$ & $\mathrm{N}$ & Proportion & $95 \% \mathrm{Cl}$ \\
\hline Smoking & 812 & & & 966 & & \\
\hline Current & 119 & 14.7 & $12.2-17.1$ & 225 & 23.3 & $20.6-26.0$ \\
\hline Past & 140 & 17.2 & $14.6-19.8$ & 496 & 51.3 & $48.2-54.5$ \\
\hline Never & 553 & 68.1 & $64.9-71.3$ & 245 & 25.4 & $22.6-28.1$ \\
\hline Sports: physically active & 347 & 42.8 & $39.4-46.2$ & 296 & 30.6 & $27.7-33.6$ \\
\hline Education years $^{\mathrm{a}}$ & 812 & & & 967 & & \\
\hline$\leq 10$ & 121 & 14.9 & $12.4-17.4$ & 36 & 3.7 & $2.5-4.9$ \\
\hline $11-13$ & 388 & 47.8 & $44.3-51.2$ & 391 & 40.4 & $37.3-43.5$ \\
\hline $14-17$ & 231 & 28.4 & $25.3-31.6$ & 335 & 34.6 & $31.6-37.6$ \\
\hline$\geq 18$ & 72 & 8.9 & $6.9-10.8$ & 205 & 21.2 & $18.6-23.8$ \\
\hline \multicolumn{7}{|l|}{ Drug use } \\
\hline Beta-blockers & 279 & 34.4 & $31.1-37.6$ & 307 & 31.7 & $28.8-34.7$ \\
\hline Anti-arrhythmics & 5 & 0.6 & $0.1-1.2$ & 8 & 0.8 & $0.3-1.4$ \\
\hline ACE-inhibitors & 254 & 31.3 & $28.1-34.5$ & 340 & 35.2 & $32.1-38.2$ \\
\hline Diuretics & 80 & 9.9 & $7.8-11.9$ & 104 & 10.8 & $8.8-12.7$ \\
\hline Ca-channel blockers & 123 & 15.1 & $12.7-17.6$ & 148 & 15.3 & $13.0-17.6$ \\
\hline \multicolumn{7}{|l|}{ Disease prevalence } \\
\hline Myocardial infarction & 20 & 2.4 & $0.8-3.7$ & 50 & 5.2 & $2.7-7.6$ \\
\hline Stroke & 27 & 3.3 & $2.1-4.6$ & 42 & 4.3 & $3.1-5.6$ \\
\hline Cardiovascular disease $^{\mathrm{b}}$ & 48 & 5.9 & $4.3-7.5$ & 153 & 15.8 & $13.5-18.1$ \\
\hline Hypertension ${ }^{c}$ & 611 & 75.2 & $72.3-78.2$ & 794 & 82.1 & $79.7-84.5$ \\
\hline Diabetes mellitus ${ }^{d}$ & 120 & 14.8 & $12.3-17.2$ & 154 & 15.9 & $13.6-18.3$ \\
\hline
\end{tabular}

SBP systolic blood pressure, DBP diastolic blood pressure, HDL high density lipoprotein cholesterol, LDL low density lipoprotein cholesterol, HbA1c glycated hemoglobin, ACE angiotensin converting enzyme, Ca-channel blockers calcium-channel blockers

${ }^{a}$ Education (years of training) according to ISCED classification 1997

${ }^{b}$ CVD: including prevalent myocardial infarction, coronary artery bypass graft (CABG), percutaneous transluminal coronary angioplasty (PTCA), stroke,

carotid surgery

${ }^{C}$ Hypertension defined as SBP equal or above 140 and/or DBP equal or above $90 \mathrm{mmHg}$, and/or use of antihypertensive medication

${ }^{\mathrm{d}}$ Diabetes defined as self-reported physician-diagnosed diabetes mellitus and/or use of anti-diabetic medication 


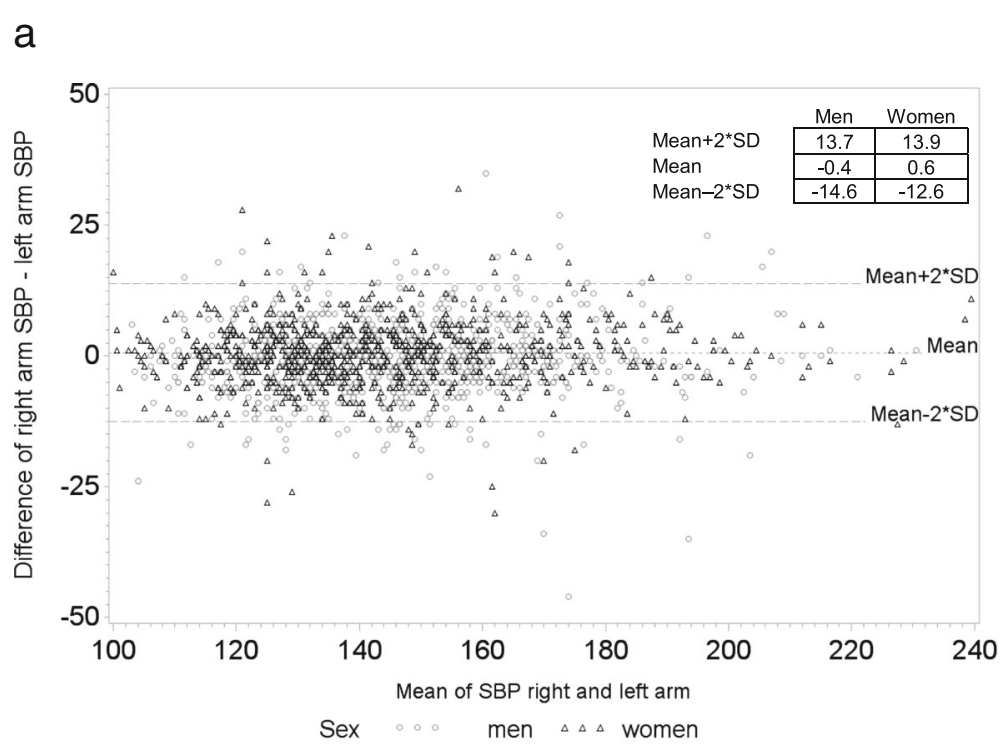

b

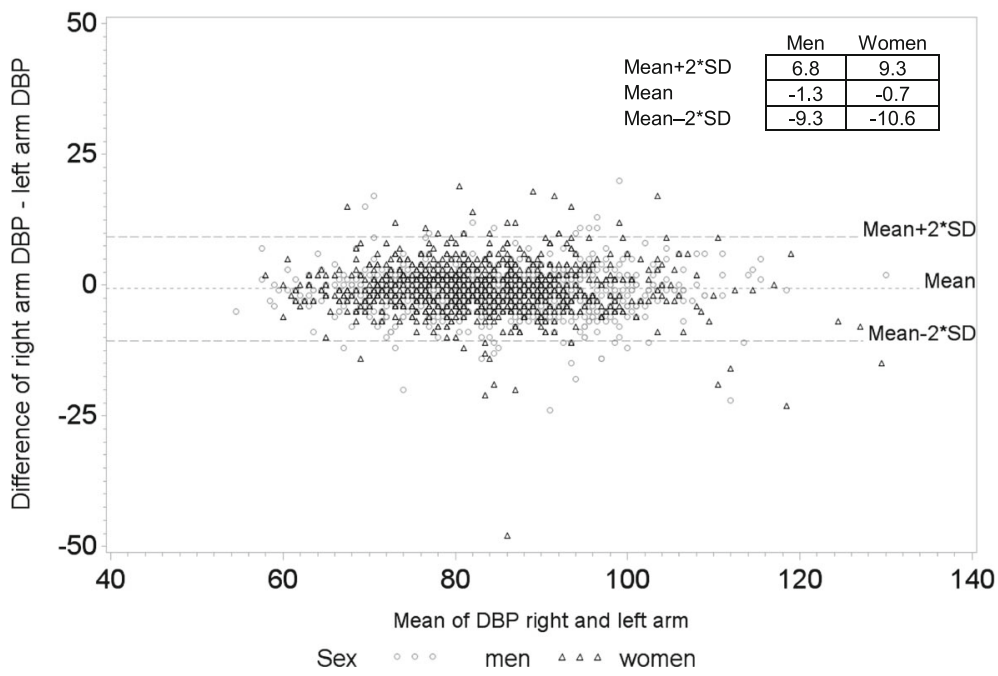

Fig. $1 \mathrm{BP}$ arm differences in measurement 4 (supine left and right arm simultaneously). BP differences between right and left arm (right - left) over mean of BP right and left arm for a systolic blood pressure (SBP) and $\mathbf{b}$ diastolic blood pressure (DBP). Black triangles represent women and grey circles men. Mean value (dotted line) and mean values \pm 2 standard deviations (SD) (long dash lines) are depicted as horizontal lines

in sitting (Figure 2). Participants had in average $1.64 \mathrm{mmHg}$ greater SBP $(95 \% \mathrm{CI}=1.20$ to 2.08$)$ and $0.51 \mathrm{mmHg}$ greater DBP $(95 \% \mathrm{CI}=0.27$ to 0.74 ) in the first sitting measurement. About $20 \%$ of the study participants had differences greater than $10 \mathrm{mmHg}$ for SBP and also $20 \%$ had differences greater than $5 \mathrm{mmHg}$ for DBP.

Figure 3 shows the adjusted means with 95\% CI for differences in BP in consecutive measurements for men and women respectively. For SBP there are clear differences in sitting position for men and women between first and second and slightly less between second and third measurements, with up to mean differences of $2 \mathrm{mmHg}$ for women between first and second measures.
Very similar patterns could be seen for SBP measurements in supine position, with greater differences for women than for men and decreasing in size with further measurements. For DBP, there are also clear differences in sitting position for men and women between first and second and less between second and third measurements, with up to almost $1 \mathrm{mmHg}$ in average for women between first and second measures. However, differences between right and left arm DBP measurements were higher for men, with differences above $1 \mathrm{mmHg}$. DBP measurements in supine position showed similar patterns for men and women, such that there is a decrease in size with further measurements. The only significant 

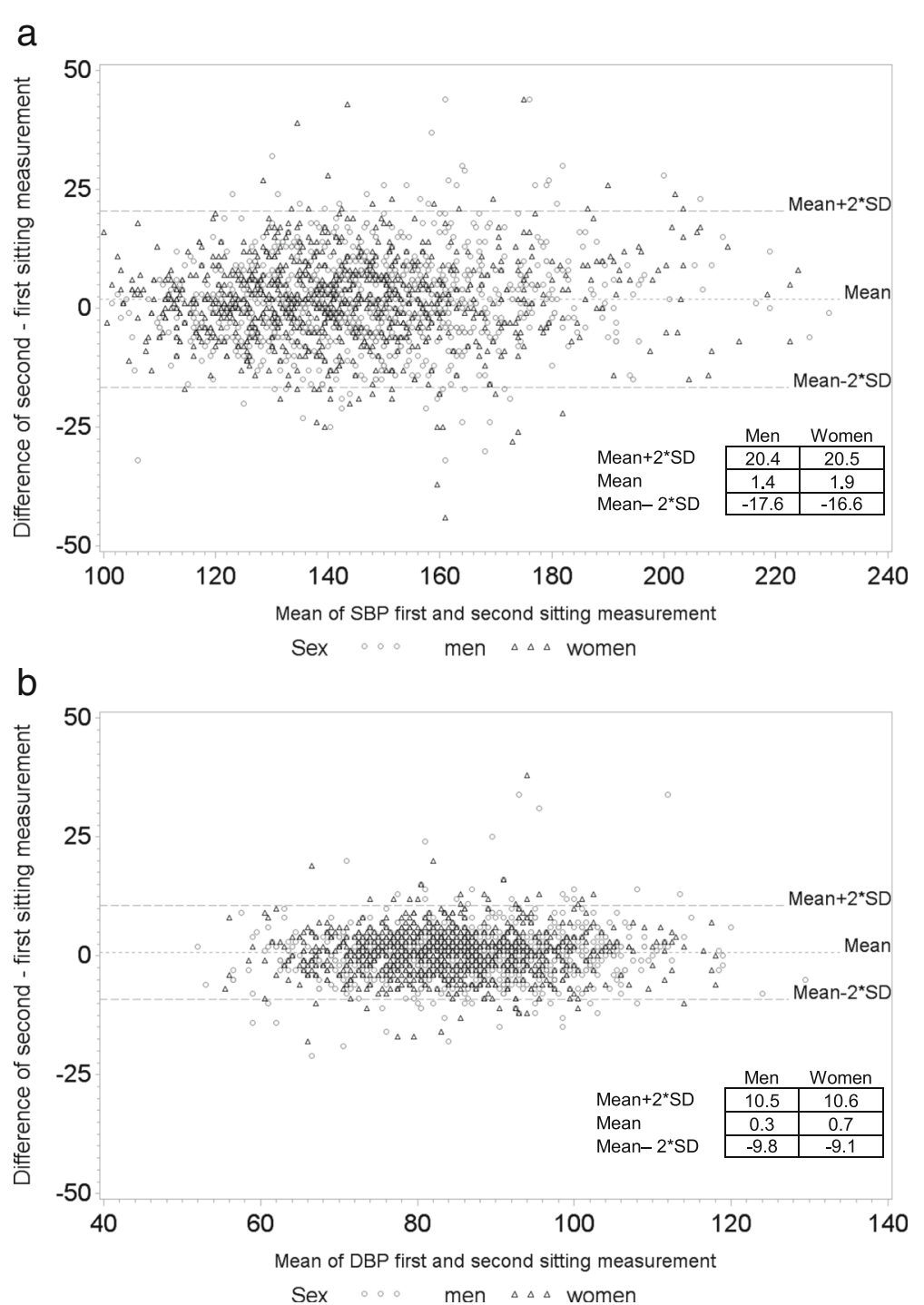

Fig. 2 BP successive measurements differences (measurement 1 vs 2). BP differences first and second sitting measurement (first - second) over mean of BP first and second sitting measurement for a systolic blood pressure (SBP) and $\mathbf{b}$ diastolic blood pressure (DBP). Black triangles represent women and grey circles men. Mean value (dotted line) and mean values \pm 2 standard deviations (SD) (long dash lines) are depicted as horizontal lines

sex difference was seen in the supine measurements were for men there was a decrease in SBP and DBP between second and third measurement, whereas for women no significant decrease could be seen based on the confidence intervals.

Table 2 shows the prevalence of hypertension when considering different BP measurements for men and women. As expected, men had higher prevalence of hypertension irrespective of BP measurement. Consecutive measurements were associated with lower hypertension rates, as SBP and DBP values diminished with further measurements. Supine measurements produced lower hypertension rates. The fact that some participants were classified as hypertensive in each individual measurement, but not with the mean of both measurement (see for example all prevalence estimations for averaged values for men, which are below individual measurement prevalence estimates), can be explained with the fact that in one measurement SBP and in the other DBP determined the hypertensive status, but after averaging both values, none of them were above 140/90 $\mathrm{mmHg}$.

\section{Discussion}

The present study sought to quantify the variability in measured BP associated with several factors (arm, position and repetition) and the consequences of body position and successive measurements on the estimation of hypertension prevalence. The main findings are that 1 ) overall, no mean BP differences were measured according to arm-side; 2) substantial higher DBP and for men also SBP 

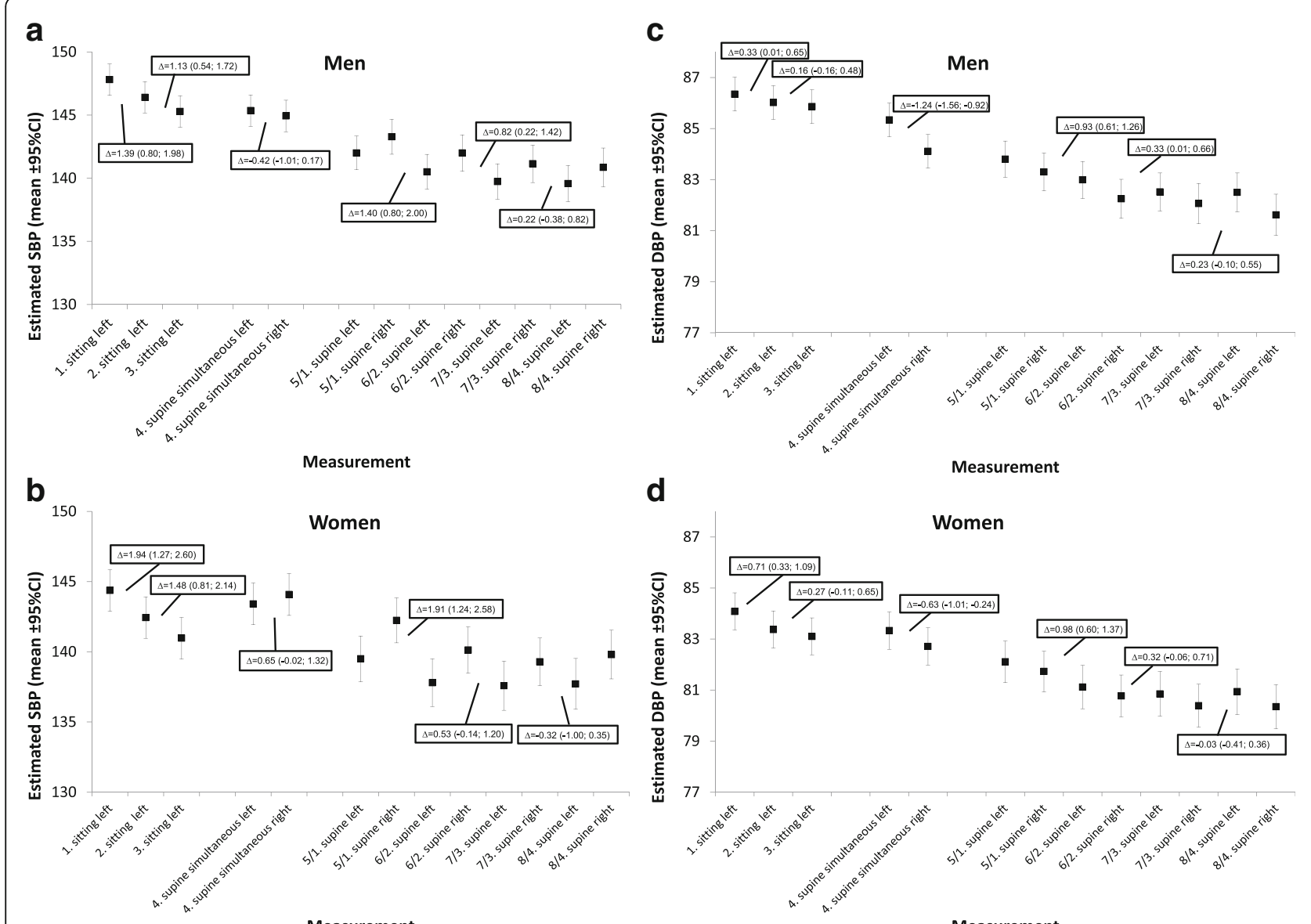

Measurement

Measurement

Fig. 3 Estimated BP by measurement irrespective of arm-side. Values presented from $5^{\text {th }}$ to $8^{\text {th }}$ measurement are the difference between the mean of one set of right and left measurements and the next set of right and left measurements. Estimated BP with $95 \% \mathrm{Cl}$ and changes in mean estimated BP across measurements for a systolic blood pressure (SBP) in men; $\mathbf{b}$ SBP in women; $\mathbf{c}$ diastolic blood pressure (DBP) in men; and $\mathbf{d}$ DBP in women. $\Delta=$ difference of the estimated BP measurements; $\mathrm{Cl}=$ confidence interval

Table 2 Prevalence of hypertension by different BP measurements, sex-stratified

\begin{tabular}{|c|c|c|c|c|c|c|}
\hline & Men & & & Women & & \\
\hline \multicolumn{7}{|l|}{ Sitting (left arm) } \\
\hline \multirow[t]{2}{*}{$1^{\text {st }}$ measurement } & $68.7 \%(N=664)$ & & & $55.3 \%(N=535)$ & & \\
\hline & & Mean $1^{\text {st }}$ and $2^{\text {nd }}$ & $66.5 \%(N=643)$ & & Mean $1^{\text {st }}$ and $2^{\text {nd }}$ & $51.7 \%(N=500)$ \\
\hline \multirow[t]{2}{*}{$2^{\text {nd }}$ measurement } & $66.8 \%(N=646)$ & & & $51.2 \%(N=495)$ & & \\
\hline & & Mean $2^{\text {nd }}$ and $3^{\text {rd }}$ & $63.2 \%(N=611)$ & & Mean $2^{\text {nd }}$ and $3^{\text {rd }}$ & $49.4 \%(N=478)$ \\
\hline $3^{\text {rd }}$ measurement & $64.0 \%(N=619)$ & & & $50.7 \%(N=490)$ & & \\
\hline \multicolumn{7}{|c|}{ Supine (arm with higher blood pressure) } \\
\hline \multirow[t]{2}{*}{$1^{\text {st }}$ measurement } & $57.5 \%(N=467)$ & & & $47.9 \%(N=389)$ & & \\
\hline & & Mean $1^{\text {st }}$ and $2^{\text {nd }}$ & $53.9 \%(N=438)$ & & Mean $1^{\text {st }}$ and $2^{\text {nd }}$ & $45.6 \%(N=370)$ \\
\hline \multirow[t]{2}{*}{$2^{\text {nd }}$ measurement } & $55.2 \%(N=448)$ & & & $44.2 \%(N=359)$ & & \\
\hline & & Mean $2^{\text {nd }}$ and $3^{\text {rd }}$ & $51.2 \%(N=416)$ & & Mean $2^{\text {nd }}$ and $3^{\text {rd }}$ & $42.2 \%(N=343)$ \\
\hline $3^{\text {rd }}$ measurement & $51.3 \%(N=417)$ & & & $42.9 \%(N=348)$ & & \\
\hline
\end{tabular}


were measured in sitting than in supine position; 3) there was a clear BP decline by consecutive measurement and 4) accordingly, the prevalence of hypertension depends strongly on the body position and successive measurements of BP.

These results replicate previous findings concerning inter-arm differences. Overall, clinically meaningful inter-arm differences were not reproducible. Reductions of $10 \mathrm{mmHg}$ for SBP and $5 \mathrm{mmHg}$ for DBP could be considered clinically relevant [25]. Yet in approximately $10 \%$ of the study-participants the inter-arm difference for SBP was above $10 \mathrm{mmHg}$. This difference can be attributable to random variation but it also could indicate that those subjects suffered from a cardiac disease, e.g. coarcation of the aorta, upper extremity arterial obstruction, dissection or aneurysm of the thoracic aorta $[10,12,19,26]$, and thus the inter-arm difference could mask treatment effects. Unfortunately, this hypothesis cannot be tested, as this accurate diagnostic information is not available for the study population. Nevertheless, in a population-based cohort and a patient-based cohort, it has been shown that there was no association between short-term blood pressure variability and subclinical target organ damage $[27,28]$.

The substantially higher DBP in sitting than in supine position is in agreement with some previous studies $[6,16]$. The finding of higher SBP in sitting than in supine position however, dissents with those studies $[6,16]$. This disagreement could be due to different protocols used in the studies mentioned above, i.e. same arm-position for measurements in sitting and supine position could lead to lower differences among readings. A further influencing factor could be the measurement order, which in our study was fixed starting with participants in sitting position. Thus the position effect cannot be separated from the successive measurements effect.

The number of readings was shown to have systematically affected the BP measurement. Compared to previous studies, we found a smaller decline from first to second sitting measurement but a more consistent decline to the third measurement [29]. There were no further reductions after the third measurement in supine (almost no difference between third and fourth measurements); thus, it seems that at that point stability was achieved. There was a similar fall in the estimated prevalence of hypertension. This decline occurred regardless of adjustment for confounders which showed to be related to the BP decline: baseline BP level, age and BMI [29]. Analysis with further adjustment for those confounders led to almost identical results (data not shown). The first measurement clearly surpassed following BP measurements. This fact could be due to a combination of white coat hypertension, blood pressure variability and/ or device accuracy [30]. Thus, the use of a single measurement introduces a systematic error that could be corrected by excluding the first reading. Accordingly, BP estimates in studies obtained from several readings are usually lower than those obtained from a single reading $[6,29,31]$. In the clinical praxis, the uncertainty about the true $\mathrm{BP}$ values has as a consequence fluctuating treatment decisions [32]. Therefore, guidelines for clinical practice recommend diagnosing hypertension based on multiple measurements at different visits. For epidemiological research, there are several difficulties (i.e. logistic and financial) associated with two-visit BP measurements, which lead to a single visit strategy. On the other hand, single visit measurements may lead to erroneous conclusions in the interpretation of prevalence rates, awareness, and medical control of hypertension in different countries [33-35]. Interestingly, it has been shown that misclassification was more common in younger $(<30$ years $)$ than older participants [36], with rates varying from $12 \%$ in subjects aged $62 \pm 11$ years [37], to $35 \%$ in subjects aged $39 \pm 9$ years and 15 to 69 years [36, 38], respectively.

There is evidence that an increased visit-to-visit variability in SBP is a powerful predictor of end-organ damage and cognitive decline [39-41]. Moreover, patients with controlled mean BP but high visit-to-visit SBP variability had an increased risk of stroke than those with low SBP variability [42]. Previous studies have shown visit-to-visit SBP variability to be strongly influenced by drug-class effects with increased variability in participants medicated with $\beta$-blockers [43]. We tried to replicate these findings with short-term SBP variability, in stratified models as well as in models with further adjustment for medication as covariate (Additional file 2: Table S1) and found no effect of medication use or medication class ( $\beta$-blockers or Angiotensin converting enzyme (ACE) inhibitors) related to SBP or DBP variability. $\mathrm{BP}$ variability results from extrinsic and intrinsic regulatory mechanisms and substantially differs in clinical significance and prognostic implications depending on the time interval considered for its assessment [44].

It has been speculated that the lack of standardization in BP measurement procedure can lead to clinically relevant differences in estimations of prevalence and incidence of hypertension [45]. Thus, it is possible that it also influences the estimates of association studies between hypertension and other factors. Therefore, published prevalence of hypertension should clearly be accompanied by a definition of the measurement procedure: arm-side, body position, number of readings and its combination for the definition of hypertension.

The present study has several important strengths. Foremost, it is a population-based sample, with welldefined health outcomes. The results of this study may be considered representative for the general population aged 45 to 85 years since a random sample from the 
population registry of the city of Halle had been selected, and a high participation rate could be achieved. Some limitations, however, need to be addressed. Due to practicability reasons, as this study was framed in the examination part of an epidemiological cohort, there were some restrictions to BP measurements: 1) there was only one simultaneous supine measurement; 2) supine measurements were not consistently throughout on one arm, but in that with higher BP. This was done following the recommendation for clinical BP measurement, that in case of disparity, the arm with higher pressure should be used for subsequent readings.

Moreover, there might be problems to separate posture effect from measurement effect. We approached this problem by including all measurements in a common statistical model with proper adjustment for measurements within the same subject. However, not all combinations of factors are observed (actually constituting an incomplete block design) and we have to rely on model extrapolations for some of the reported differences. Thus slight differences measured in sitting vs supine positions (mean difference for SBP was $4.05 \mathrm{mmHg}$ and for DBP was $2.98 \mathrm{mmHg}$ greater in sitting) could be due to successive measurements instead of body position.

A further question is how much of the BP variability can be due to the measuring device. It has been reported that the OMRON HEM-705CP device fulfils the recommendation criteria of the international protocol [46]. Finally, different resting times were used for sitting and supine readings ( 3 and 1 min respectively).

\section{Conclusions}

In conclusion, the measured BP strongly depends on position, successive measurements and combination of subsequent BP readings. Thus, BP measurements should only be compared between studies applying equal measurement conditions. Moreover, the first BP measurement should not be used to define hypertension since it overestimates mean individual BP. The mean of second and third measurement offers the advantage of better reproducibility over single measurements.

\section{Additional files}

Additional file 1: Figure S1. Flow chart of the study design. (PPT $176 \mathrm{~kb}$ ) Additional file 2: Table S1. Estimated BP by measurement stratified by medication use. (DOCX $19 \mathrm{~kb})$

\section{Abbreviations}

ACE: Angiotensin converting enzyme; ARMA: Autoregressive moving-average; BP: Blood pressure; Ca-channel blockers: Calcium-channel blockers; Cl: Confidence interval; CVD: Cardiovascular disease; DBP: Diastolic blood pressure; HbA1c: Glycated hemoglobin; HDL: High density lipoprotein cholesterol; LDL: Low density lipoprotein cholesterol; SBP: Systolic blood pressure

\section{Acknowledgements}

We thank all participants of the CARLA study and all members of the CARLA study team who participated in the recruitment, data collection, data management, and analysis. We are also indebted to numerous colleagues who shared their instruments of data collection with us and gave advice and practical help during the design phase and implementation of the recruitment and examination.

\section{Funding}

Deutsche Forschungsgemeinschaft as part of the Collaborative Research Centre 598 'Heart failure in the elderly - cellular mechanisms and therapy'; Wilhelm-Roux Programme of the Martin Luther University of HalleWittenberg (FKZ 14/41, 16/19); Federal Employment Office, and Ministry of Education and Cultural Affairs of Saxony-Anhalt (MK-CARLA-MLU-2011).

\section{Availability of data and materials}

All CARLA-data are stored at Institute of medical epidemiology, biostatistic and informatics, Martin-Luther University Halle-Wittenberg, and are available upon request.

\section{Authors' contributions}

AK, OK, DT, DM, SN, KHG, SF, JH conceived the design of the study; MEL and AK conducted the data analysis with assistance from OK. AK drafted the manuscript. MEL edited the manuscript. All authors contributed to the manuscript preparation and approved the final version manuscript.

\section{Competing interests}

The authors declare that they have no competing interests.

\section{Consent for publication}

Not applicable.

\section{Ethics approval and consent to participate}

The study was in accordance with the declaration of Helsinki. All participants gave their written informed consent. The study was approved by the local ethic commission at the Medical Faculty of the Martin-Luther-University Halle-Wittenberg.

\section{Author details}

${ }^{1}$ Institute of Medical Epidemiology, Biostatistics and Informatics, Martin-Luther University Halle-Wittenberg, Magdeburger Str. 8, 06112 Halle Saale, Germany. ${ }^{2}$ Centre for Health and Society, Faculty of Medicine, Heinrich Heine University Düsseldorf, Düsseldorf, Germany. ${ }^{3}$ Institute for Biometrics and Epidemiology, German Diabetes Center, Leibniz Institute for Diabetes Research at Heinrich Heine University Düsseldorf, Düsseldorf, Germany. ${ }^{4}$ Department of Medicine III, Martin-Luther University Halle-Wittenberg, Halle Saale, Germany. ${ }^{5}$ German Cancer Research Centre, Division of Cancer Epidemiology, Heidelberg, Germany.

Received: 25 May 2016 Accepted: 6 January 2017

Published online: 18 January 2017

\section{References}

1. Pearce KA, Grimm Jr RH, Rao S, Svendsen K, Liebson PR, Neaton JD, Ensrud K. Population-derived comparisons of ambulatory and office blood pressures. Implications for the determination of usual blood pressure and the concept of white coat hypertension. Arch Intern Med. 1992;152(4):750-6.

2. Kawahara J, Sano H, Fukuzaki H, Saito K, Hirouchi H. Acute effects of exposure to cold on blood pressure, platelet function and sympathetic nervous activity in humans. Am J Hypertens. 1989;2(9):724-6.

3. Campbell NR, McKay DW, Chockalingam A, Fodor JG. Errors in assessment of blood pressure: sphygmomanometers and blood pressure cuffs. Can J Public Health. 1994:85 Suppl 2:S22-25.

4. Handler J. The importance of accurate blood pressure measurement. Perm J. 2009:13(3):51-4.

5. Gould BA, Hornung RS, Kieso HA, Altman DG, Raftery EB. Is the blood pressure the same in both arms? Clin Cardiol. 1985;8(8):423-6.

6. Jamieson MJ, Webster J, Philips S, Jeffers TA, Scott AK, Robb OJ, Lovell HG, Petrie JC. The measurement of blood pressure: sitting or supine, once or twice? J Hypertens. 1990;8(7):635-40. 
7. Netea RT, Lenders JW, Smits P, Thien T. Both body and arm position significantly influence blood pressure measurement. J Hum Hypertens. 2003;17(7):459-62

8. Tolonen H, Koponen P, Naska A, Mannisto S, Broda G, Palosaari T, Kuulasmaa K, Project EP. Challenges in standardization of blood pressure measurement at the population level. BMC Med Res Methodol. 2015;15:33.

9. Cassidy $\mathrm{P}$, Jones K. A study of inter-arm blood pressure differences in primary care. J Hum Hypertens. 2001;15(8):519-22.

10. Lane D, Beevers M, Barnes N, Bourne J, John A, Malins S, Beevers DG. Interarm differences in blood pressure: when are they clinically significant? J Hypertens. 2002;20(6):1089-95.

11. O'Shea JC, Murphy MB. Ambulatory blood pressure monitoring: which arm? J Hum Hypertens. 2000;14(4):227-30.

12. Singer AJ, Hollander JE. Blood pressure. Assessment of interarm differences. Arch Intern Med. 1996;156(17):2005-8.

13. Clark CE, Powell RJ. The differential blood pressure sign in general practice: prevalence and prognostic value. Fam Pract. 2002;19(5):439-41.

14. Orme S, Ralph SG, Birchall A, Lawson-Matthew P, McLean K, Channer KS. The normal range for inter-arm differences in blood pressure. Age Ageing 1999;28(6):537-42.

15. Kristensen $\mathrm{BO}$, Kornerup $\mathrm{HJ}$. Which arm to measure the blood pressure? Acta Med Scand Suppl. 1982;670:69-73.

16. Netea RT, Smits $P$, Lenders JW, Thien T. Does it matter whether blood pressure measurements are taken with subjects sitting or supine? J Hypertens. 1998;16(3):263-8.

17. Netea RT, Lenders JW, Smits P, Thien T. Influence of body and arm position on blood pressure readings: an overview. J Hypertens. 2003;21(2):237-41.

18. Terent A, Breig-Asberg E. Epidemiological perspective of body position and arm level in blood pressure measurement. Blood Press. 1994;3(3):156-63.

19. Pickering TG, Hall JE, Appel LJ, Falkner BE, Graves J, Hill MN, Jones DW, Kurtz T, Sheps SG, Roccella EJ. Recommendations for blood pressure measurement in humans and experimental animals: part 1: blood pressure measurement in humans: a statement for professionals from the Subcommittee of Professional and Public Education of the American Heart Association Council on High Blood Pressure Research. Circulation. 2005;111(5):697-716.

20. Hay M. Sex, the brain and hypertension: brain oestrogen receptors and high blood pressure risk factors. Clin Sci (Lond). 2016;130(1):9-18.

21. Greiser KH, Kluttig A, Schumann B, Kors JA, Swenne CA, Kuss O, Werdan K, Haerting J. Cardiovascular disease, risk factors and heart rate variability in the elderly general population: design and objectives of the CARdiovascular disease, Living and Ageing in Halle (CARLA) Study. BMCCardiovascDisord. 2005;5:33.

22. Roger VL, Go AS, Lloyd-Jones DM, Benjamin EJ, Berry JD, Borden WB, Bravata DM, Dai S, Ford ES, Fox CS, et al. Heart disease and stroke statistics2012 update: a report from the American Heart Association. Circulation. 2012;125(1):e2-e220

23. Brown HP R. Applied Mixed Models in Medicine. 2nd ed. Chichester England: John Wiley \& Sons Ltd; 2006

24. Law MR, Morris JK, Wald NJ. Use of blood pressure lowering drugs in the prevention of cardiovascular disease: meta-analysis of 147 randomised trials in the context of expectations from prospective epidemiological studies. BMJ. 2009;338:b1665.

25. Staessen JA, Wang J. Blood-pressure lowering for the secondary prevention of stroke. Lancet. 2001;358(9287):1026-7.

26. Eguchi K, Yacoub M, Jhalani J, Gerin W, Schwartz JE, Pickering TG Consistency of blood pressure differences between the left and right arms. Arch Intern Med. 2007;167(4):388-93.

27. Madden JM, O'Flynn AM, Dolan E, Fitzgerald AP, Kearney PM. Short-term blood pressure variability over $24 \mathrm{~h}$ and target organ damage in middleaged men and women. J Hum Hypertens. 2015;29(12):719-25.

28. Wittke El, Fuchs SC, Moreira LB, Foppa M, Fuchs FD, Gus M. Blood pressure variability in controlled and uncontrolled blood pressure and its association with left ventricular hypertrophy and diastolic function. J Hum Hypertens. 2016;30(8):483-7.

29. Schulze MB, Kroke A, Saracci $R$, Boeing $H$. The effect of differences in measurement procedure on the comparability of blood pressure estimates in multi-centre studies. Blood Press Monit. 2002;7(2):95-104.

30. Pater C. Beyond the Evidence of the New Hypertension Guidelines. Blood pressure measurement - is it good enough for accurate diagnosis of hypertension? Time might be in, for a paradigm shift (I). Curr Control Trials Cardiovasc Med. 2005;6(1):6.
31. Wietlisbach V, Rickenbach M, Burnand B, Hausser D, Gutzwiller F. Combining repeated blood pressure measurements to obtain prevalences of high blood pressure. Acta Med Scand Suppl. 1988;728:165-8.

32. Kerr EA, Zikmund-Fisher BJ, Klamerus ML, Subramanian U, Hogan MM, Hofer TP. The role of clinical uncertainty in treatment decisions for diabetic patients with uncontrolled blood pressure. Ann Intern Med. 2008;148(10):717-27.

33. Birkett NJ. The effect of alternative criteria for hypertension on estimates of prevalence and control. J Hypertens. 1997;15(3):237-44.

34. Modesti PA, Agostoni P, Agyemang C, Basu S, Benetos A, Cappuccio FP, Ceriello A, Del Prato S, Kalyesubula R, O'Brien E, et al. Cardiovascular risk assessment in low-resource settings: a consensus document of the European Society of Hypertension Working Group on Hypertension and Cardiovascular Risk in Low Resource Settings. J Hypertens. 2014;32(5): 951-60.

35. Modesti PA, Rapi S. From the Epidemiologist to the Physician: A Complex Issue for a Labile Risk. J Clin Hypertens. 2015;17(6):428-30.

36. Modesti PA, Rapi S, Bamoshmoosh M, Baldereschi M, Massetti L, Padeletti L, Gensini GF, Zhao D, Al-Hidabi D, Al Goshae H. Impact of one or two visits strategy on hypertension burden estimation in HYDY, a population-based cross-sectional study: implications for healthcare resource allocation decision making. BMJ Open 2012, 2(4). doi:10.1136/bmjopen-2012-001062.

37. Figueiredo D, Azevedo A, Pereira M, de Barros H. Definition of hypertension: the impact of number of visits for blood pressure measurement. Rev Port Cardiol. 2009;28(7-8):775-83.

38. Lang T, de Gaudemaris R, Chatellier G, Hamici L, Diene E. Epidemiology Group of the Societe Francaise d'Hypertension A, the IWPG: Prevalence and therapeutic control of hypertension in 30,000 subjects in the workplace. Hypertension. 2001;38(3):449-54.

39. Cuffe RL, Howard SC, Algra A, Warlow CP, Rothwell PM. Medium-term variability of blood pressure and potential underdiagnosis of hypertension in patients with previous transient ischemic attack or minor stroke. Stroke. 2006:37(11):2776-83.

40. Howard SC, Rothwell PM. Reproducibility of measures of visit-to-visit variability in blood pressure after transient ischaemic attack or minor stroke. Cerebrovasc Dis. 2009;28(4):331-40.

41. Howard SC, Rothwell PM. Cerebrovascular Cohort Studies C: Regression dilution of systolic and diastolic blood pressure in patients with established cerebrovascular disease. J Clin Epidemiol. 2003;56(11):1084-91.

42. Rothwell PM, Howard SC, Dolan E, O'Brien E, Dobson JE, Dahlof B, Sever PS, Poulter NR. Prognostic significance of visit-to-visit variability, maximum systolic blood pressure, and episodic hypertension. Lancet. 2010;375(9718): 895-905.

43. Rothwell PM, Howard SC, Dolan E, O'Brien E, Dobson JE, Dahlof B, Poulter NR, Sever PS, Ascot B, Investigators MRCT. Effects of beta blockers and calcium-channel blockers on within-individual variability in blood pressure and risk of stroke. Lancet Neurol. 2010;9(5):469-80.

44. Parati G, Ochoa JE, Salvi P, Lombardi C, Bilo G. Prognostic value of blood pressure variability and average blood pressure levels in patients with hypertension and diabetes. Diabetes Care. 2013;36 Suppl 2:S312-324.

45. Magalhaes MG, Oliveira LM, Christofaro DG, Ritti-Dias RM. Prevalence of high blood pressure in Brazilian adolescents and quality of the employed methodological procedures: systematic review. Rev Bras Epidemiol. 2013;16(4):849-59.

46. El Assaad MA, Topouchian JA, Asmar RG. Evaluation of two devices for selfmeasurement of blood pressure according to the international protocol: the Omron M5-I and the Omron 705IT. Blood Press Monit. 2003:8(3):127-33. 